\title{
Report
}

\section{Performance of the Resonant Schottky pickup at CSRe}

\author{
J. X. Wu ${ }^{\mathrm{a}}$, Y. D. Zang ${ }^{\mathrm{a}}$, F. Nolden ${ }^{\mathrm{b}}$, M. S. Sanjari ${ }^{\mathrm{b}}$, P. Hülsmann ${ }^{\mathrm{b}}$, F. Caspers ${ }^{\mathrm{c}}$, T. C.

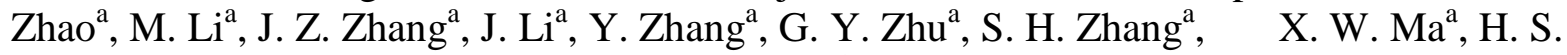 \\ $\mathrm{Xu}^{\mathrm{a}}$, J. C. Yang ${ }^{\mathrm{a}}$, J. W. Xia ${ }^{\mathrm{a}}$, R. S. Mao ${ }^{\mathrm{a}}$, P. Petri ${ }^{\mathrm{b}}$ \\ ${ }^{a}$ Institute of Modern Physics, Chinese Academy of Sciences, Lanzhou, China \\ ${ }^{\mathrm{b}}$ Gesellschaft fur Schwerionenforschung, Darmstadt, Germany \\ ${ }^{\mathrm{c}}$ CERN, Geneva Switzerland
}

Keywords: CSRe, Schottky signal, Resonant Schottky pickup

\begin{abstract}
For a bunched beam, the signals on pickups are coherent, providing a signal power proportional to the square of the number of particles. For a coasting beam the individual particle signals have a random phase; therefore, the overall signal is proportional to the particle number $\mathrm{N}$. As a consequence, Schottky signals are often relatively weak and have to compete with many noise sources. For a small number of particles, the $\mathrm{S} / \mathrm{N}$ is always a problem. To achieve a high signal-to-noise ratio and better temporal resolution, which could yield important physical information about fast processes, a resonant pickup was developed at GSI ${ }^{[1]}$, and a similar device is now installed in the CSRe ${ }^{[2]}$ at IMP. This device will be used for nuclear mass and lifetime measurement, as well as other uses. The final goal of the pickup is to detect single particles. In Dec 2012, we performed an experiment with a ${ }^{112} \mathrm{Sn}^{50+}$ beam with an energy of $253 \mathrm{MeV} / \mathrm{u}$, and the single particle sensitivity of the pickup was successfully confirmed. This paper presents hardware measurements of the pickup as well as beam measurements.
\end{abstract}




\section{Performance of the Resonant Schottky pickup at CSRe}

J. X. Wu ${ }^{\mathrm{a}}$, Y. D. Zang ${ }^{\mathrm{a}}$, F. Nolden ${ }^{\mathrm{b}}$, M. S. Sanjari ${ }^{\mathrm{b}}$, P. Hülsmann ${ }^{\mathrm{b}}$, F. Caspers ${ }^{\mathrm{c}}$, T. C. Zhao ${ }^{\mathrm{a}}$, M. Li ${ }^{\mathrm{a}}$, J. Z. Zhang ${ }^{\mathrm{a}}$, J. Li ${ }^{\mathrm{a}}$, Y. Zhang ${ }^{\mathrm{a}}$, G. Y. Zhu ${ }^{\mathrm{a}}$, S. H. Zhang,

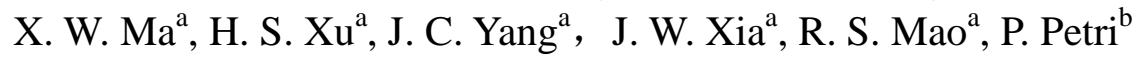

${ }^{a}$ Institute of Modern Physics, Chinese Academy of Sciences, Lanzhou, China

${ }^{\mathrm{b}}$ Gesellschaft fur Schwerionenforschung, Darmstadt, Germany

${ }^{\mathrm{c}}$ CERN, Geneva Switzerland

\section{Abstract}

For a bunched beam, the signals on pickups are coherent, providing a signal power proportional to the square of the number of particles. For a coasting beam the individual particle signals have a random phase; therefore, the overall signal is proportional to the particle number $\mathrm{N}$. As a consequence, Schottky signals are often relatively weak and have to compete with many noise sources. For a small number of particles, the S/N is always a problem. To achieve a high signal-to-noise ratio and better temporal resolution, which could yield important physical information about fast processes, a resonant pickup was developed at GSI ${ }^{[1]}$, and a similar device is now installed in the $\mathrm{CSRe}^{[2]}$ at IMP. This device will be used for nuclear mass and lifetime measurement, as well as other uses. The final goal of the pickup is to detect single particles. In Dec 2012, we performed an experiment with a ${ }^{112} \mathrm{Sn}^{50+}$ beam with an energy of $253 \mathrm{MeV} / \mathrm{u}$, and the single particle sensitivity of the pickup was successfully confirmed. This paper presents hardware measurements of the pickup as well as beam measurements.

Key Words: CSRe, Schottky signal, Resonant Schottky pickup

\section{Introduction}

HIRFL-CSR is a multipurpose cooling storage ring system that consists of a main ring (CSRm), an experimental ring (CSRe) and a radioactive beam line (RIBLL2). The two cyclotrons SFC and SSC are used as its injectors. Many physics experiments are performed on HIRFL-CSR, and one of the most important techniques used is Schottky mass spectroscopy, which is used to measure unknown masses and lifetimes of exotic nuclides. Such measurements require very high sensitivity for detections in systems where single or few particle beams could be identified. For that purpose, a non-destructive resonant pickup was investigated and mounted on CSRe.

The pickup installed on CSRe (see Fig. 1) is a pillbox cavity; Fig. 2 shows its MAFIA model in two dimensions for the simulation, where the beam moves along the z-axis. The two copper plunger pistons, shown on the top of the cavity in Fig. 1, are controlled by two step motors to adjust the resonant frequency in a range of $\pm 2 \mathrm{MHz}$ to match all CSRe beam energies. The radius of the cavity is $0.3 \mathrm{~m}$, and the length is $0.11 \mathrm{~m}$. The vacuum tube has a radius of $0.125 \mathrm{~m}$ (see Fig. 2). The ceramic pipe (colored in dark blue in Fig. 2) with a thickness of $9.5 \mathrm{~mm}$ and a length of $67 \mathrm{~mm}$ in the beam direction is used to form a gap and to seal the vacuum tube. The ceramic holder and the vacuum tube, shown in red in Fig. 2, are made of stainless steel. The pillbox itself is made of stainless steel as well, but the inner surface is coated with a copper layer approximately $50 \mu \mathrm{m}$ thick, which is well above the skin depth at the resonant frequency. The cavity is cut into two halves in the beam direction in case the pickup is not needed and can be taken apart from the ceramic gap, for example to avoid the parasitic impedances that might be harmful for intense circulating beams or for vacuum baking. The two half-cylinders are electrically connected (by copper contact springs) to the beam pipe forming a closed resonant volume around the ceramic tube. In the lower part of the pickup, the signal is coupled out via a coupling loop, which is mounted on a flange. A detailed description of the design and construction of the pickup can be found in reference [1]. 


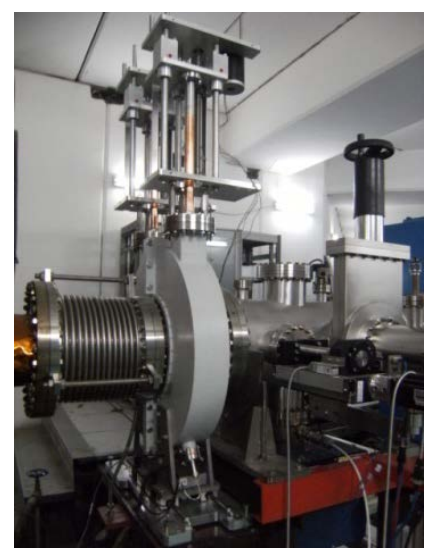

Figure 1: Resonant Schottky pickup installed on CSRe. The cavity in light blue is mounted on a sliding carriage to facilitate easy removal or installation of the two half-cylinders. On top of the cavity, two frequency tuners are mounted on two flanges. The two copper plunger pistons can be remotely controlled to move inside the resonator to adjust the frequency. The signal output is installed in the lower part of the cavity.

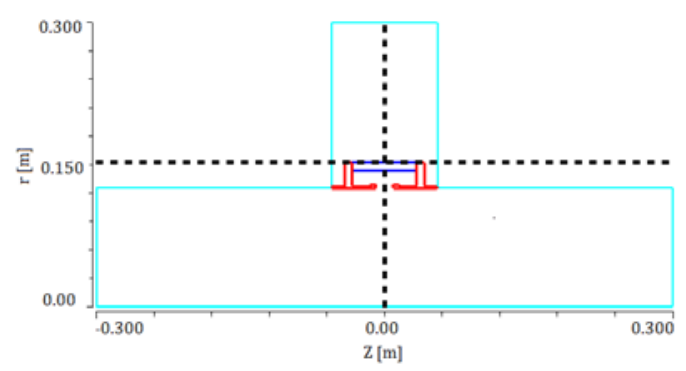

Figure 2: 2d plot of the pickup for the simulation with MAFIA. The ceramic gap is shown in dark blue, while the ceramic supporter and part of the vacuum tube are shown in red.

\section{Simulation and lab measurement of the pickup}

\section{Resonant frequency and $Q$ value}

From a simple-minded estimate without considering the ceramic inside, the resonant frequency of a cylindrical cavity in the $\mathrm{TM}_{010}$ mode would be $\mathrm{f}=\mathrm{C} * 2.405 /(\mathrm{R} * 2 * \pi)=382 \mathrm{MHz}$, and the $\mathrm{Q}$ value would be approximately 22500. However, from the simulation with MAFIA and with the ceramic inserted, the result is much different. If the permittivity of the ceramic is set to $\varepsilon=9.6-j * 9.6 * 0.0005=9.6-j * 0.0048$, i.e., the dielectric absorption of the ceramic is taken into account, using the eigenmode solver the frequency of the first mode $\left(\mathrm{TM}_{010}\right)$ is $f=2.237 \cdot 10^{8} \mathrm{~Hz}+j \cdot 1.830 \cdot 10^{4} \mathrm{~Hz}$, and the dielectric power loss is

$$
P_{\text {diel }}=\frac{2 \pi \operatorname{Re}(f) W}{Q_{\text {diel }}}=\frac{2 \pi \operatorname{Re}(f) W}{\operatorname{Re}(f) / 2 \operatorname{Im}(f)}=2.0747 \cdot 10^{-6} \mathrm{~W}
$$

where $\mathrm{W}$ is the energy stored in the cavity, and $Q_{\text {diel }}$ is the quality factor resulting from the power loss in the lossy dielectric material.

If all the surface materials are made of copper with a conductance of $5.8 \cdot 10^{7} \mathrm{~S} / \mathrm{m}$, the power loss due to the induced wall currents is $4.75 \cdot 10^{-7} \mathrm{~W}$. However, if all the stainless steel parts (red color in Fig. 2) with conductivity $1.33 \cdot 10^{6} \mathrm{~S} / \mathrm{m}$ are taken into account, the power loss is increased to $1.12 \cdot 10^{-6} \mathrm{~W}$ and the unloaded $Q$ value is

$$
Q=\frac{P_{\text {wall }}+P_{\text {diel }}}{\omega W}=1940
$$

which is much lower than the theoretical value, mainly due to dielectric losses.

The reflection measurement of the resonant pickup with a network analyzer is shown in Fig. 3. From this measurement, the resonant frequency is $242.36585 \mathrm{MHz}$, the unloaded $\mathrm{Q}$ value ${ }^{[3]}$ is $\mathrm{Q}=979$, and the loaded $\mathrm{Q}$ value 
is $Q_{L}=496.8$. Thus, the real $Q$ value is even less than the simulated value. This could be because of inaccurate simulation of the power loss of the cavity. Inaccuracies might be caused by neglecting the surface roughness of the metal or because of the imperfect contact between the cavity and the tube, which may have led to field leakage and have increased the effective surface resistance, or other similar factors.

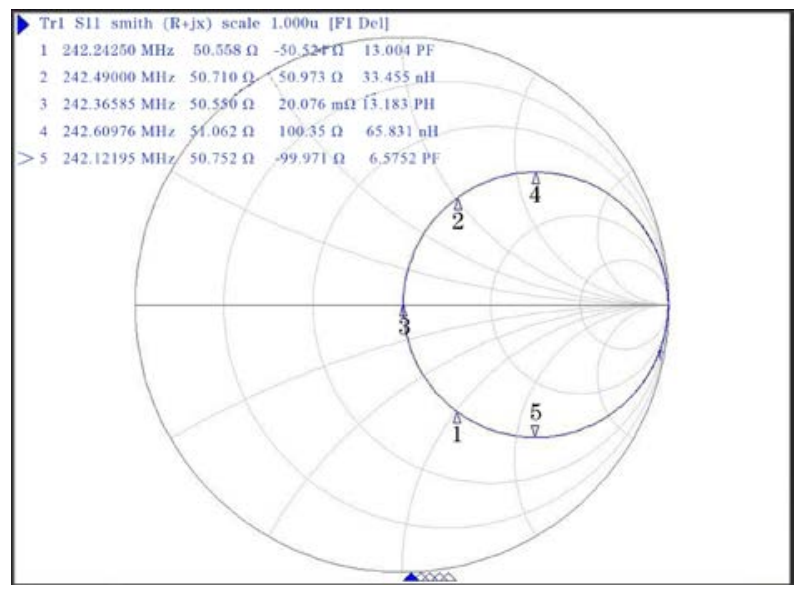

Figure 3: Reflection measurement of the resonant pickup (Center frequency: $242 \mathrm{MHz}$, Span: $10 \mathrm{MHz}$ )

\section{2. $\mathbf{R}_{\mathrm{s}} / \mathrm{Q}$ measurement}

The $\mathrm{R}_{\mathrm{s}} / \mathrm{Q}$ value, which is a measure for providing an accelerating voltage $\mathrm{V}$ (peak voltage) with a certain stored energy $\mathrm{W}$, is one of the key parameters to characterize cavity resonators:

$$
\frac{R_{s}}{Q}=\frac{V^{2}}{4 \pi f_{0} W}=\frac{\left(\int E(z, z(t)) \cdot d z\right)^{2}}{4 \pi f_{0} W}=\frac{1}{4 \pi f_{0} W}\left|\int E_{z}(z) e^{j 2 \pi f z(\beta c)} \cdot d z\right|^{2}=\frac{R_{s}^{\prime} T^{2}}{Q}
$$

where $R_{s}^{\prime}$ is the shunt impedance, and $T=\frac{\int E_{z}(z) \cos \left(\frac{\Omega z}{\beta c}\right) d z}{\int E_{z}(z) d z}$ is the transit time factor. $R_{s}^{\prime} / Q$ depends only on the mode geometry and is actually a measure of the "efficiency" of the cavity as a kicker. From the reciprocity theorem, it is also a measure of the "ability" to detect a beam as a pickup.

Thus, if one wants to determine $R_{s}^{\prime} / Q$, the field distribution must be known. To measure the field distribution in a resonant cavity, one usually uses a perturbation method: a small perturbing object is moved along some path through the cavity, and the resonance frequency is measured as a function of the position of the object. We used a hollow copper cylinder with outer diameter $D_{0}=4 \mathrm{~mm}$, inner diameter $\mathrm{D}_{\mathrm{i}}=2 \mathrm{~mm}$ and length $2 \mathrm{l}=14 \mathrm{~mm}$, which was controlled by a step motor to move along the longitudinal axis of the resonator. The result is shown in Fig. 4. Because the resonant frequency also varies with temperature ${ }^{[4]}$, as can be clearly observed in Fig. 4, the frequency shift related to the temperature change should be corrected. Here a simple correction was performed. The whole process was divided into three sections: two sections outside of the electric field area of the cavity and one section inside the field area. The whole frequency shift from temperature change in each section was averaged to every measurement step; the corrected frequency shift is shown by the red curve in Fig. 4. 


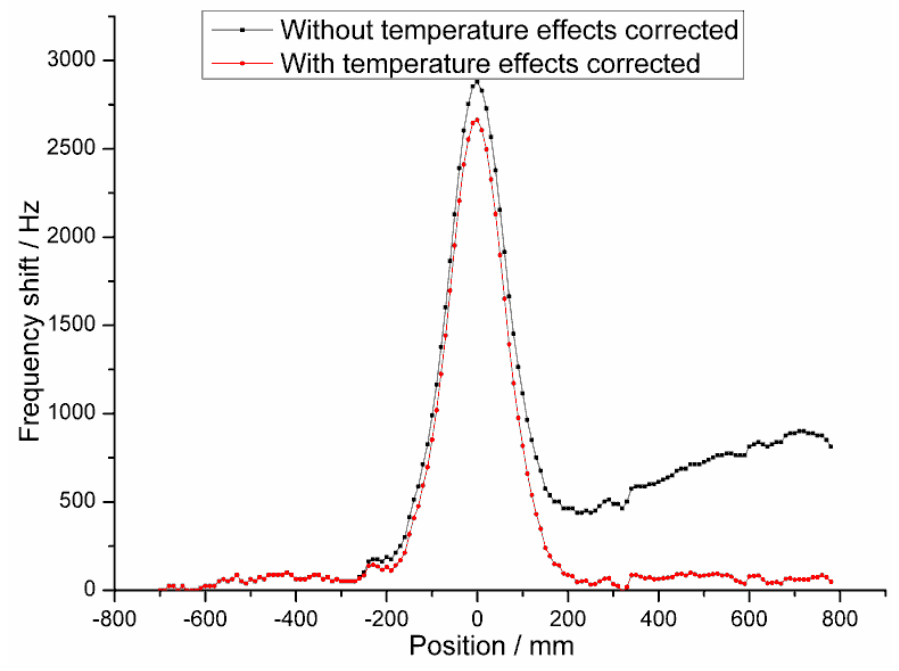

Figure 4: Frequency shift as a function of the position of the perturbing object

The presence of the perturbing object produces a local distortion of the field distribution in the region around it. After correcting for the longitudinal and the transverse components of $\mathrm{E}$ and $\mathrm{H}$ with the depolarization of the cylindrical perturbing object considered, the frequency shift of the cavity is given by ${ }^{[5]}$

$$
\frac{\Delta f}{f_{0}}=\frac{1}{W}\left(-K_{1} \varepsilon_{0} E_{\square}^{2}-K_{2} \varepsilon_{0} E_{\perp}^{2}+K_{3} \mu_{0} H_{\square}^{2}+K_{4} \mu_{0} H_{\perp}^{2}\right)
$$

The coefficients K1 to K4 are proportional to the electric and magnetic polarizability of the perturbing object. For a metallic cylinder perturbing along the longitudinal axis of the cavity, K1 is much larger than K2, K3 and K4, and the frequency shift is then essentially due to the component of the longitudinal electric field $E_{z}\left(E_{z}=E_{\square}\right)$.

$$
\begin{gathered}
-\frac{\Delta f}{f_{0}}=\frac{K_{1} \varepsilon_{0} E_{\square}^{2}}{W} \\
K_{1}=\frac{\pi}{3} \cdot l^{3} \cdot\left[\arg \operatorname{sh}\left(\frac{2}{3 \pi} \frac{l}{D_{0} / 2}\right)\right]^{-1}
\end{gathered}
$$

The electric field is then

$$
E_{z}=\left(\frac{W}{K_{1} \varepsilon_{0}}\left|\frac{\Delta f}{f_{0}}\right|\right)^{1 / 2}
$$

Thus the electric field distribution can be known from the frequency shift. By combining eq. 3 and eq. 7, we obtain

$$
\frac{R_{s}^{\prime}}{Q}=\left.\left.\frac{1}{4 \pi f_{0}^{2} K_{1} \varepsilon_{0}}\left|\int\right| \Delta f(z)\right|^{1 / 2} \cdot d z\right|^{2}
$$

Therefore, from the measured frequency shift we can determine the $R_{s}^{\prime} / Q$ value, which is $51.8 \Omega$.

A long thin cylindrical ceramic rod was also used to measure $R_{s}^{\prime} / Q$ by placing it so that its long axis coincided with the longitudinal axis of the resonator. If the rod is aligned parallel to the electric field and inserted inside the cavity, the frequency shift is ${ }^{[6]}$

$$
-\frac{\Delta f}{f_{0}}=\frac{\varepsilon_{0}\left(1-\varepsilon_{r}\right) \pi r^{2} \int\left|E_{z}\right|^{2} \cdot d z}{4 W}
$$

where $\mathrm{r}$ is the rod radius and $\varepsilon_{r}$ is the relative permittivity of the ceramic rod. The expression of $R_{s}^{\prime} / Q$ is then

$$
\frac{R_{s}^{\prime}}{Q}=\frac{\left(\int E_{z} \cdot d z\right)^{2}}{4 \pi f_{0} W}=\frac{1}{\pi^{2} r^{2}} \cdot \frac{\Delta f}{f_{0}^{2}} \cdot \frac{\left|\int E_{z}(z) \cdot d z\right|^{2}}{\int\left|E_{z}\right|^{2} \cdot d z} \cdot \frac{1}{\varepsilon_{0}\left(1-\varepsilon_{r}\right)}=\frac{1}{\pi^{2}} \cdot \frac{1}{\varepsilon_{0}\left(1-\varepsilon_{r}\right)} \cdot \frac{L}{r^{2}} \cdot \frac{\Delta f}{f_{0}^{2}}
$$

where $\mathrm{L}$ is the geometric averaged rod length. A ceramic rod with a diameter of $3 \mathrm{~mm}$ was used in the 
measurement, and $R_{s}^{\prime}$ / $Q$ turns out to be $48.1 \Omega$, which is close to the measurement with the small hollow copper cylinder.

\section{Beam test result for the Schottky resonant pickup}

To reduce the effects of noise and interference, the coupled signal of the resonant Schottky pickup is directly sent to a low noise preamplifier with a gain of $27 \mathrm{~dB}$, frequency range of $0.1-2 \mathrm{GHz}$ and noise figure of $0.7 \mathrm{~dB}$. Then, the signal is bandpass filtered to prevent noise overload of the next two $40 \mathrm{~dB}$ amplifiers. A second $40 \mathrm{~dB}$ amplifier can be optionally added for very low signals before the signal is transmitted over $20 \mathrm{~m}$ of high quality coaxial cable to a commercial digital spectrum analyzer. With three amplifiers, two filters and approximately $25 \mathrm{~m}$ of coaxial cable, the total transmission gain at the resonant working frequency is approximately 102-104 dB.

The first experiment with resonant Schottky pickup was performed in Dec 2011 with a ${ }^{22} \mathrm{Ne}^{10+}$ beam at 70 $\mathrm{MeV} / \mathrm{u}$ and with electron cooling on. While using the electron-cooled beam, the sensitivity of the pickup was calibrated with currents measured by a DC current transformer. For the purpose of single particle detection we performed another experiment in Dec 2012. The measurements from both experiments are described in the following.

\section{Calibration and $R_{s} / Q$ measurement}

A DC current transformer was used as for calibration of the Schottky power, which is shown in Fig. 5. The linear fit yields a relation between the Schottky power at the spectrum analyzer input port and the beam current $\mathrm{P}$ $(\mathrm{mW})=0.09307 * \mathrm{I}_{\mathrm{B}}(\mu \mathrm{A})$ for a ${ }^{22} \mathrm{Ne}^{10+}$ beam, $70 \mathrm{MeV} / \mathrm{u}$.

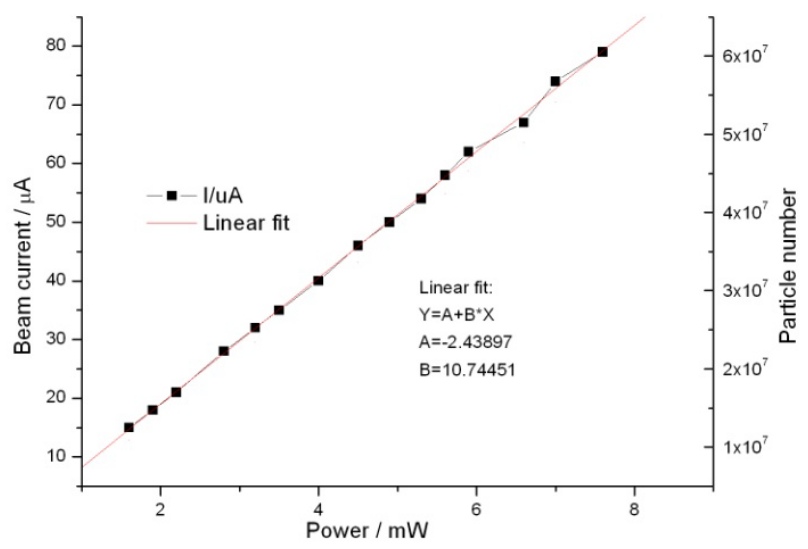

Figure 5: Measured Schottky power as a function of the beam current/particle number

From reference [1], the output power of the pickup for each harmonic can be expressed as

$$
P_{p}=N\left(q e f_{0}\right)^{2} \frac{R_{S}}{Q} Q_{L}
$$

For a ${ }^{22} \mathrm{Ne}^{10+}$ beam with an energy of $70 \mathrm{MeV} / \mathrm{u}$ circulating in CSRe, the revolution frequency is $0.855 \mathrm{MHz}$ and $\mathrm{R}_{\mathrm{s}} / \mathrm{Q}$ would be $7.51 \Omega$ with a transmission gain of $102.58 \mathrm{~dB}$ at the measurement frequency. From the measured field distribution, when $\beta$ is 0.3673 , T will be 0.36729 , and thus $R_{S}^{\prime} / Q=55.7 \Omega$ which is not too different from the forward rod and small cylinder measurements.

\section{Single particle detection}

In Dec 2012, one single particle detection was successfully performed with the resonant Schottky pickup for a ${ }^{112} \mathrm{Sn}^{50+}$ beam. The primary beam extracted from the CSRm synchrotron was ${ }^{112} \mathrm{Sn}^{35+}$ with an energy of 376.42 $\mathrm{MeV} / \mathrm{u}$. After the target (Be target, $10 \mathrm{~mm}$ thick) on the radioactive beam transmission line, the secondary beam was ${ }^{112} \mathrm{Sn}^{50+}$ with an energy of $252.923 \mathrm{MeV} / \mathrm{u}$, which was thereafter injected into CSRe with electron cooling off. The machine was set in an isochronous operation mode to a ${ }^{94} \mathrm{Ru}^{44+}$ beam with $\gamma=1.302, \mathrm{~B} \rho=5.5294$ instead of $\gamma=1.271$. So for ${ }^{112} \mathrm{Sn}^{50+}$ beam emerging from the target, the momentum dispersion is large, which can be seen in Fig. 6 where the frequency width is up to $25 \mathrm{kHz}$. To achieve single particle detection, the intensity of 
the injected beam to CSRe was slowly decreased until sometimes there was signal on the resonant pickup and sometimes nothing except noise (see Fig. 7 and Fig. 8). This experimental condition was confirmed by the TOF detector, which can recognize single particles accurately. Both detectors measured exactly the same probability for either having particles in the storage ring or not. In the figures, the black trace was the memory trace for the case with no beam inside the ring, i.e., the noise trace. It can be observed clearly on the spectrogram in Fig. 7 that the beam was lost and there was no beam inside the ring anymore. In Fig. 8 the channel band power with beam is $-21.14 \mathrm{dBm}$ and from Fig. 7 the noise band power is $-21.19 \mathrm{dBm}$, so the net signal power is $P=\left(10^{-21.14 / 10}-10^{-21.19 / 10}\right) \cdot 1 \mathrm{~mW}=88 \mathrm{nW}$. From the measurement results of $\mathrm{R}_{\mathrm{s}} / \mathrm{Q}$ and the signal transmission gain, the power arriving on the spectrum analyzer due to a signal particle can be estimated for ${ }^{112} \mathrm{Sn}^{50+}$ beam with an energy of $252.923 \mathrm{MeV} / \mathrm{u}$ : approximately $26.6 \mathrm{nW}$. Thus, there are three or four particles in Fig.8 but at the right side of the trace, there can only be one particle. Because of the noisy background it is difficult to distinguish between two or three particles, but the estimated and the measured signal power is roughly at the same level.

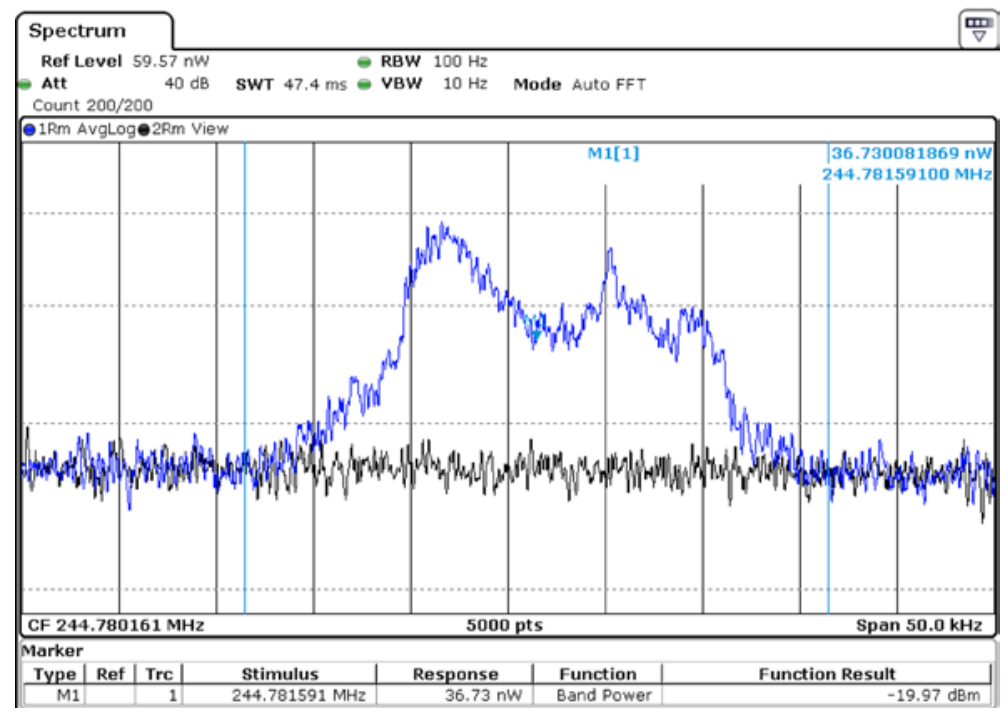

Figure 6: Schottky spectra for high intensity beam (Center frequency: $244.780161 \mathrm{MHz}$, Span: $50 \mathrm{kHz}$ ). As the electron cooling was switched off, the momentum spread of the beam was large.

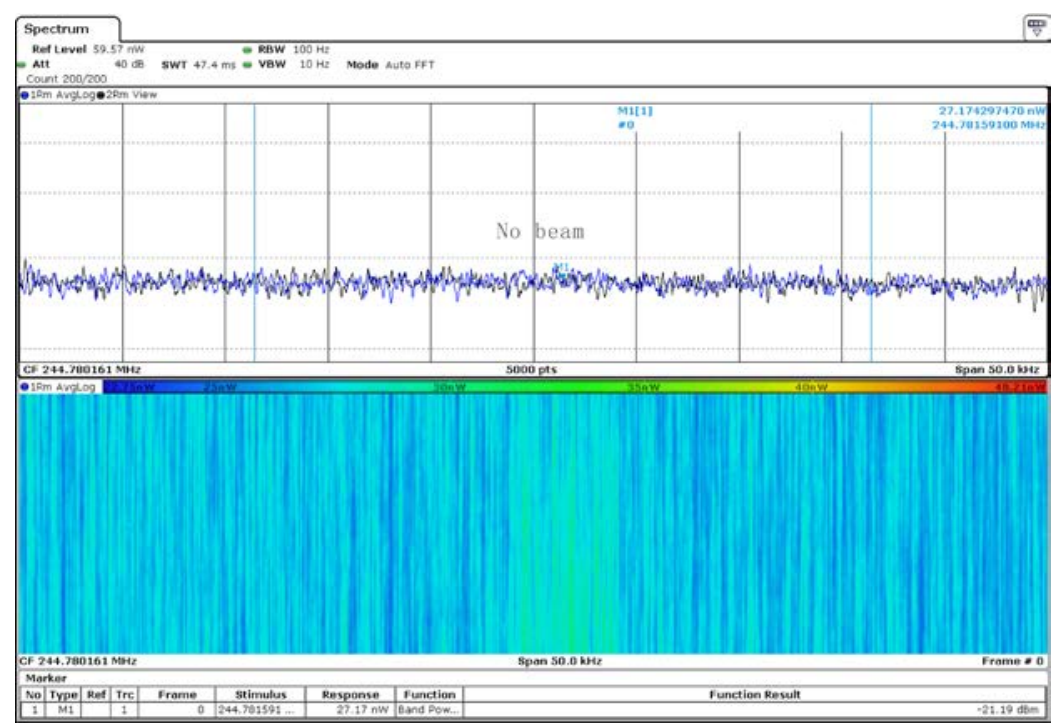

Figure 7: Schottky spectrogram when no particle is inside the ring in the end (Center frequency: $244.780161 \mathrm{MHz}$, Span: 50 kHz, RBW: 100 Hz, Band power: -21.19 dBm). 


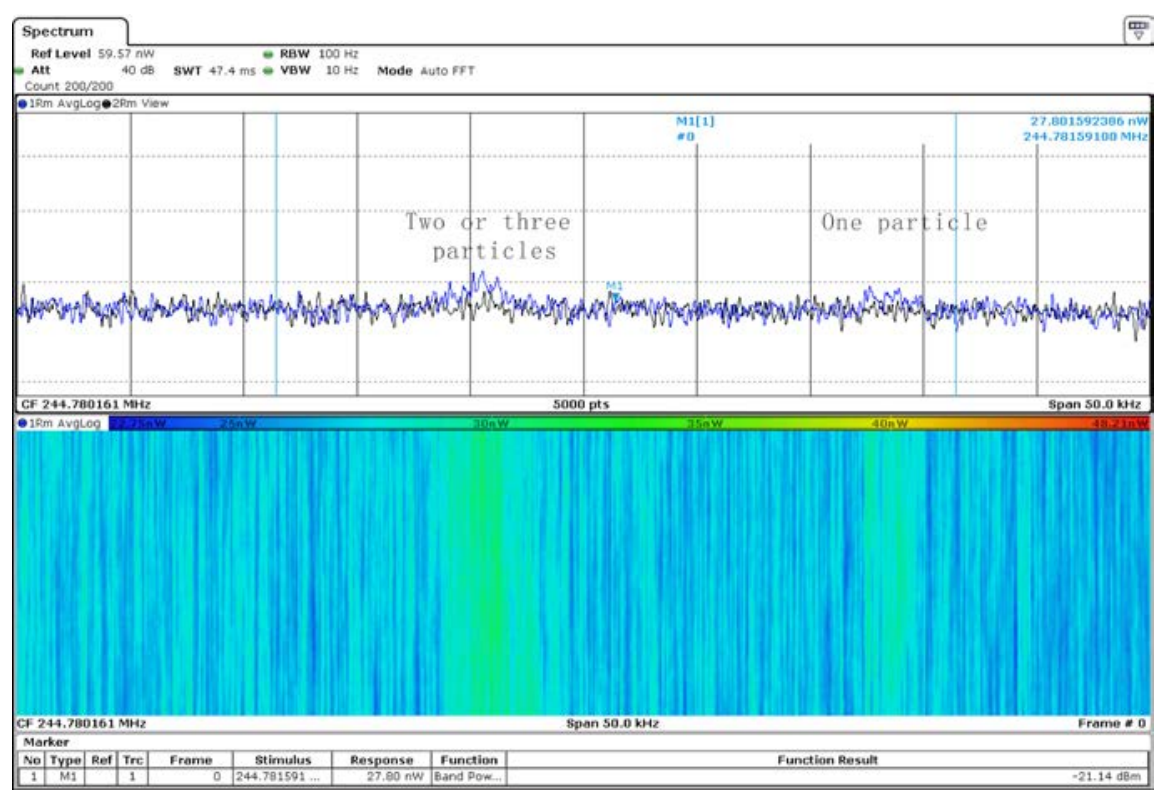

Figure 8: Schottky spectrogram for single particle detection (Center frequency: $244.780161 \mathrm{MHz}, \mathrm{Span}: 50 \mathrm{kHz}$, RBW: $100 \mathrm{~Hz}$, Band power: -21.14 dBm).

\section{Conclusion}

The resonant Schottky pickup was installed at CSRe and beam tests were performed successfully. This instrument was shown to be very sensitive and powerful, with the ability to detect even single particles. It is obvious that with the resonant pickup, we can substantially increase our experimental performance.

Using perturbation theory with the depolarization of the cylindrical perturbing object considered, the measured $R_{S}^{\prime} / Q$ is $51.8 \Omega$. The value found using the ceramic rod measurement is $48.1 \Omega$, whereas the beam measurement result is $55.7 \Omega$. The three results are in good agreement.

\section{References}

[1] F. Nolden, et al., A fast and sensitive resonant Schottky pickup for heavy ion storage rings, NIM A 659 (2011) 69-77.

[2] J. X. Xia, W. L. Zhan, B. W. Wei et al. NIM A 488 (2002) 11-25.

[3] F. Caspers, RF Engineering Basic Concepts: The Smith Chart. CAS 2010, Aarhus, June 2010.

[4] Jilong Ju, A novel configuration of temperature compensation in the resonant cavities, IEEE Trans. Microwave Theory Tech., Vol. 52, No. 1, Jan. 2004, 139-143

[5] F. Caspers, G. Dome, Precise perturbation measurements of resonant cavities and higher order mode identification, CERN-SPS/85-46 (ARF)

[6] R. G. Carter, Accuracy of microwave cavity perturbation measurements, IEEE Trans. on Microwave theory and Techniques, Vol. 49, No. 5, May 2001, pp. 918-923. 